\title{
Primary prevention of cardiovascular disease: managing hypertension and hyperlipidaemia
}

\author{
F D R Hobbs
}

Heart 2004;90(Suppl IV):iv22-iv25. doi: 10.1136/hrt.2004.037580

Cardiovascular medicine has a sound evidence base upon which health professionals can base their interventions to modify risk among the British public. For primary prevention of cardiovascular disease, however, while there is considerable evidence on what to do, data are limited on how the evidence should be implemented in practice. The challenge will be to learn by experience which interventions directed at reducing blood pressure and lipids levels work best in different settings. There is a need to structure care to identify individuals who are at risk. Current targets are explicit and achievable for both hypertension and lipids. Effective treatment is likely to require multiple drug treatment.

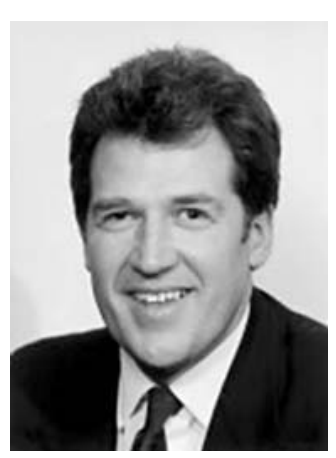

$\mathrm{T}$ he curvilinear relation between blood pressure and risk is well established. Systolic blood pressure is the most important, with a fivefold adjusted relative risk compared to a threefold adjusted relative risk for diastolic pressure (fig 1). ${ }^{1}$ There is also a curvilinear relation between serum cholesterol and mortality from coronary heart disease (CHD) (fig 2). ${ }^{2}$ However, the most important message to emerge during the past decade is that these risks factors are multiplicative. This has led to the development of systems for predicting an individual's cardiovascular risk.

Patients with type 2 diabetes are at particularly high cardiovascular risk. Haffner and colleagues reported that whatever cardiovascular outcome is looked at (myocardial infarction, stroke, or cardiovascular deaths), patients with diabetes who have not had a myocardial infarction have the same seven year incidence of cardiovascular events as patients without diabetes who have already suffered a myocardial infarction. ${ }^{3}$ These data persuaded the Americans to view diabetes as a coronary risk equivalent. In the UK, a more conservative view is taken and the National Service Framework for diabetes does not place diabetes as a coronary risk equivalent, although the revised 2004 guidelines will view diabetes as requiring secondary prevention. The true situation is probably that in people who have had diabetes for some time-established diabetic populations and those for whom there has been a delay in diagnosis-diabetes is a coronary risk equivalent. But if disease is diagnosed earlier, then it is probably an intermediate risk. This is reflected in the NSF for diabetes recommendation to intervene with a statin at a $15 \% 10$ year
University of Birmingham Birmingham B15 2TT, UK f.d.r.hobbs@bham.ac.uk
Correspondence to:
Professor F D R Hobbs Primary Care and Genera Practice, Primary Care $\ldots \ldots \ldots \ldots \ldots$ risk rather than a $30 \% 10$ year risk. It is important to remember that the explosion of diabetes is being driven by rising obesity. US data show a close association between the increase in weight and the increase in incidence of diabetes. ${ }^{4}$

It has been known for more than 10 years that intervention is needed to treat hypertension and hyperlipidaemia. A meta-analysis in 1990 of hypertension intervention trials showed a $42 \%$ risk reduction in stroke by treating hypertension and a $14 \%$ reduction in CHD. ${ }^{5}$ At one point it was thought that treating hypertension would only influence stroke; however, by pooling the data treatment was shown also to influence CHD. The interesting observation was that while the expected reduction was seen in stroke risk, the analysis did not show the $20-25 \%$ reduction in CHD that epidemiological studies had suggested. The explanation for this is almost certainly that stroke is more related to the pressor effect of blood pressure while CHD is more dependent on multifactorial risk. Until attention was also paid to reducing lipids, the expected reductions in CHD were not seen in clinical trials.

The hypertension optimal treatment (HOT) trial $^{6}$ has guided recommendations on target blood pressure. It showed that risk of a major cardiovascular event was significantly reduced if systolic pressure was reduced to below $150 \mathrm{~mm} \mathrm{Hg}$, and that optimal systolic blood pressure was $138 \mathrm{~mm} \mathrm{Hg}$. For diastolic pressure, $<90 \mathrm{~mm} \mathrm{Hg}$ was significantly better than $95 \mathrm{~mm} \mathrm{Hg}$ in reducing risk, and the optimal level was $83 \mathrm{~mm} \mathrm{Hg}$.

The HOT trial also demonstrated that targets that are historically considered to be difficult can be achieved in routine primary care practice, provided that combination therapy is used (fig 3 ). At the end of the five year trial, only $32 \%$ patients were taking a single drug, $48 \%$ of patients were taking two drugs, and $20 \%$ were taking three or four drugs.

Trials have also shown that even in high risk groups, such as patients with diabetes, an incremental benefit is achieved by more aggressive blood pressure control. In the UK prospective diabetes study (UKPDS), tight control (144/ $82 \mathrm{~mm} \mathrm{Hg}$ ) was associated with a $24 \%$ reduction in risk of cardiovascular events compared with less tight control (154/87 mm Hg). ${ }^{7}$ However, attention to blood pressure should not be at the exclusion of glycaemic control. In UKPDS, the people who had the best outcomes were those

\footnotetext{
Abbreviations: CHD, coronary heart disease; EUROASPIRE, European action on secondary prevention by intervention to reduce events; HOT, hypertension optimal treatment; MRFIT, multiple risk factor intervention trial; UKPDS, UK prospective diabetes study
} 

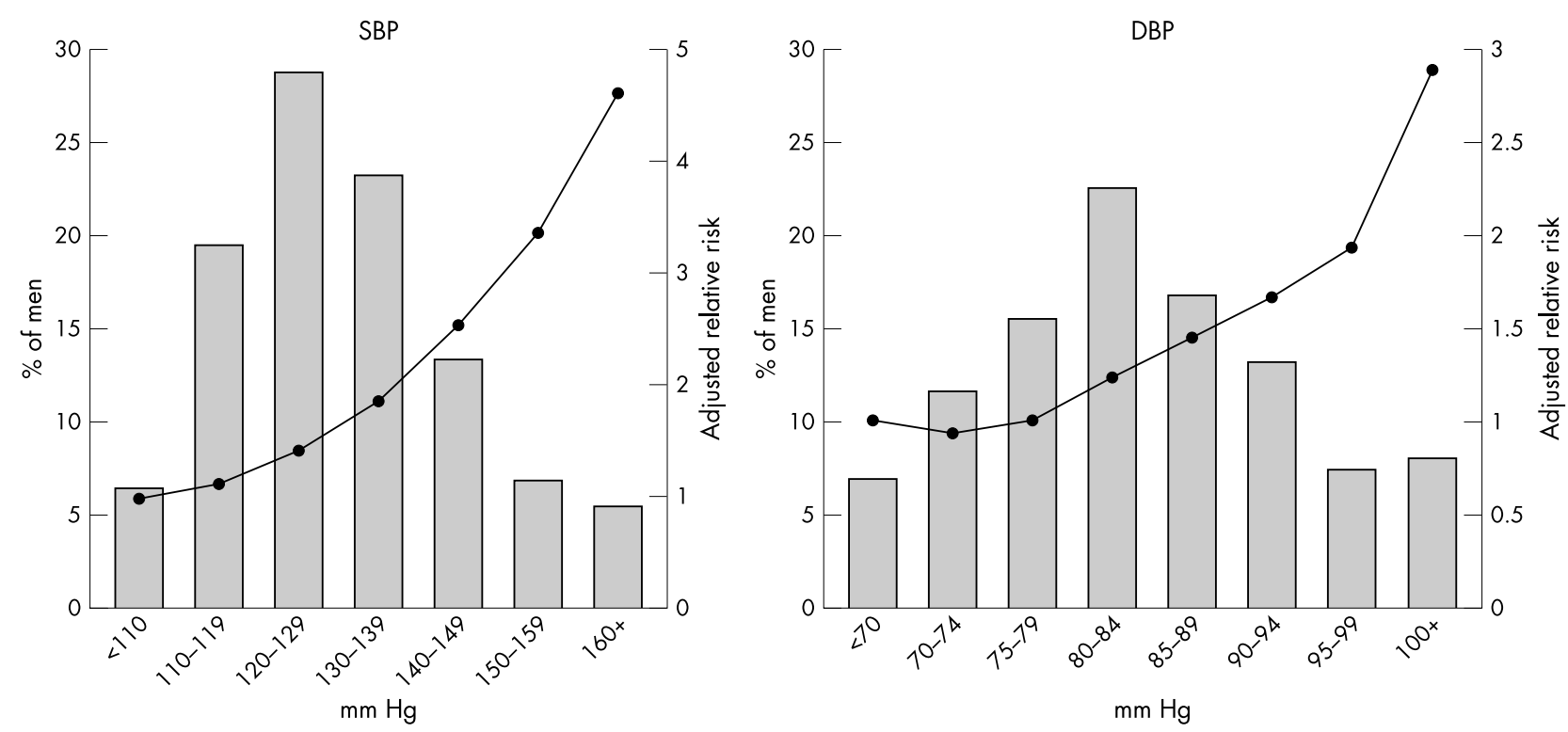

Figure 1 Adjusted relative risk of cardiovascular mortality by systolic blood pressure (SBP) and diastolic blood pressure (DBP) in men screened for the multiple risk factor intervention trial (MRFIT) (adapted with permission from National High Blood Pressure Education Program Working Group').

who had the tightest blood pressure control and also the tightest $\mathrm{HbA}_{1 \mathrm{C}}$ control. All risk factors need to be treated equally aggressively.

For lipid lowering, it is known from epidemiological studies that every $0.5 \mathrm{mmol} / \mathrm{l}$ increase in total cholesterol corresponds to an increase in CHD mortality risk of $12 \%$ and an increase in mortality risk of $17 \%$ when adjusted for regression dilution bias. $^{8}$ Furthermore, the major lipid reduction trials have shown that every $1 \mathrm{mmol} / \mathrm{l}$ reduction in total cholesterol was associated with a $25 \%$ reduction in CHD risk. ${ }^{9}$ There is also clear evidence from intervention trials that benefit of this magnitude is achieved whichever method is used to reduce cholesterol concentrations.

\section{HOW WELL IS SECONDARY PREVENTION WORKING?}

The health survey for England ${ }^{10}$ showed that in 1998 only 9.3\% of hypertensive patients in England were reaching a blood pressure target of 140/90 $\mathrm{mm} \mathrm{Hg}$. At that time doctors were working to a less aggressive target, and so the result is not as disappointing as it first appears. However, most countries have got a long way to go in terms of blood pressure

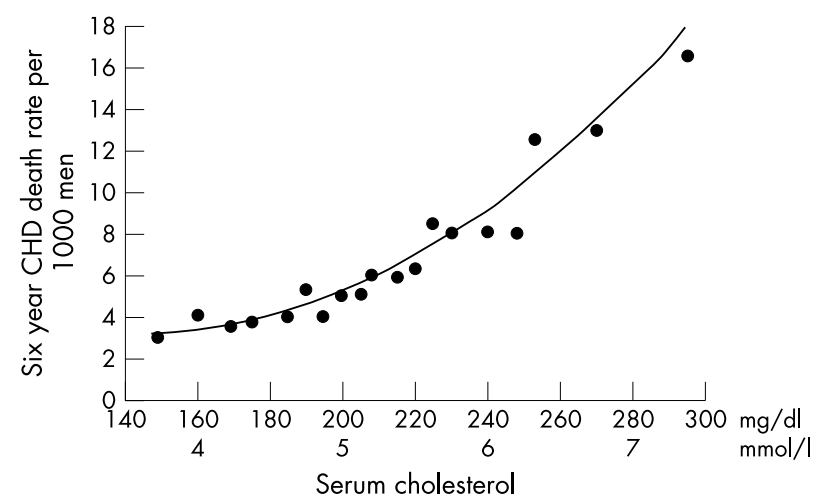

Figure 2 Age adjusted coronary heart disease (CHD) death rate and serum cholesterol in 361662 US men screened for MRFIT (adapted with permission from Martin et $a^{2}$ ). control and it is not only primary care that is failing: the EUROASPIRE surveys, from tertiary centres across Europe, involved patients discharged after a myocardial infarction or a coronary intervention. A comparison of 1995/6 and 1999/00 surveys ${ }^{11}$ shows that although there were good improvements in the proportion of patients with cholesterol values at or below target, there was little change in the proportion of patients whose high blood pressure was inadequately controlled while rates of smoking and obesity had worsened. These data represent the best resourced centres in Europe and demonstrate that it is not easy to implement even quite simple evidence.

Statins are extremely effective and are also well tolerated drugs. In clinical trials, only $6-12 \%$ of patients withdrew from treatment as a consequence of adverse events compared with about $6 \%$ on placebo. However, in routine practice much higher proportions of patients discontinue treatment. One of the major issues is therefore to help patients to stay on a treatment that is not only going to modify their mortality risk but is also going to modify their quality of life by avoiding significant events.

\section{PRIMARY PREVENTION}

The reason primary prevention of cardiovascular disease is so important is that in about $50 \%$ of cases CHD presents as acute myocardial infarction or sudden cardiac death. Delaying intervention until people present with angina-the most common manifestation of cardiovascular disease-is neither tenable nor likely to be cost effective to the health system.

The scale of the task facing health care professionals in the UK is enormous. It is impossible to say what success is being achieved in primary prevention because there are no reliable data on the denominator population. However, the health survey for England ${ }^{12}$ gives some indication of the number of people who might be eligible for primary prevention. In the 1998 survey, at least a quarter of the adult population in England had an adverse lipid profile. The best estimate is that, using a level of 30\% 10 year risk, around 15\% of the adult population in the UK is eligible for intervention. If the intervention target drops to $20 \% 10$ year risk (which it is expected to do), then nearly $20 \%$ of adults would be eligible for intervention. 
At enrolment

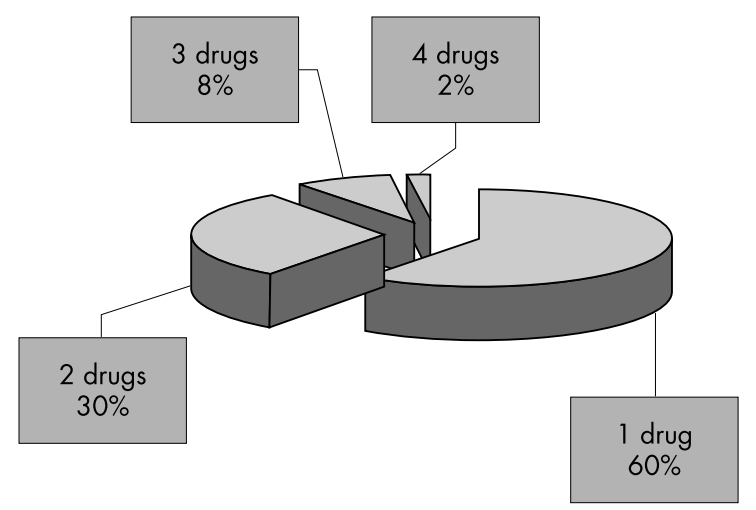

Mean $\mathrm{BP}=161 / 98 \mathrm{~mm} \mathrm{Hg}$
Final visit

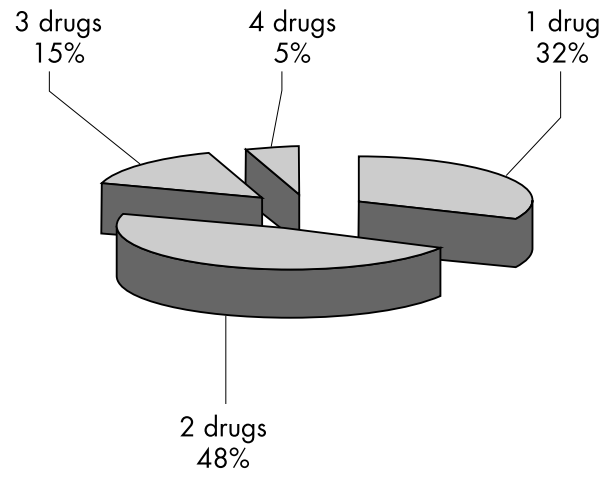

Mean BP $=142 / 83 \mathrm{~mm} \mathrm{Hg}$

Figure 3 Combination therapy was needed to achieve blood pressure targets in the HOT study. ${ }^{6}$

\section{PUBLIC PERCEPTIONS OF CARDIOVASCULAR DISEASE}

The health professions are clear about what needs to be done, but is the public? A survey in five European countries asked a random 5000 members of the general public for their opinions about cardiovascular risk. Only $45 \%$ of people surveyed correctly identified CHD as the leading cause of death in their country. ${ }^{13}$ People mistakenly believed that cancer was a greater personal risk. There were also significant gaps in knowledge about what causes cardiovascular disease.

General practitioners in the same countries were also surveyed and $92 \%$ of physicians thought that their patients would know that elevated cholesterol was associated with cardiovascular disease. ${ }^{14}$ In reality, less than half of the public recognised an association between cholesterol and elevated risk; stress was rated as more important than cholesterol. Thirty per cent did not recognise an association between smoking and CHD and only a quarter were aware of the relevance of different lipid fractions. ${ }^{13}$ There are therefore important issues to tackle relating to increasing the general public's understanding of cardiovascular disease.

\section{TASKS FACING CLINICIANS}

Tools such as the risk prediction charts, and the related computerised algorithms, act as an empirical guide to which patients should be targeted in terms of primary prevention. If a decision is made to intervene, the Joint British Societies recommendations on cardiovascular disease prevention provide explicit, relatively simple and achievable targets for both hypertension and lipids. ${ }^{15}$ The lipid targets are expected to become more aggressive in early 2004 .

There are several reasons for optimism that cardiovascular prevention will improve. It is known that offering brief advice and nicotine replacement therapy to smokers, and doing this repeatedly, will achieve success (around 15\%) in helping people to stop smoking. Screening and structured follow up clinics are known to work: for blood pressure there needs to be opportunistic screening, and lipids need to be checked in people with hypertension or diabetes, in the over 40s, and at age 18 in the case of premature cardiovascular disease in a parent (father $<55$ years, mother $<65$ years). Checks should also be made for diabetes, based on symptoms and obesity.

Major problems that need closer attention include the rising incidence of obesity, metabolic syndrome, and diabetes. Government policies on food, alcohol, smoking, and access to exercise are crucial for improving the nation's cardiovascular health.

\section{CONCLUSION}

The various national service frameworks and National Institute for Clinical Excellence guidelines legitimise cardiovascular disease as a priority, and the new General Medical Services GP contract also encourages cardiovascular disease management. There is a need to structure care to identify those at risk, and then to apply structured lifestyle and therapeutic interventions and monitoring of these patients. The call-recall systems that are applied so effectively in terms of cancer prevention need to be applied to cardiovascular primary prevention. Inevitably prescribing will rise, and there will be a need for polypharmacy for many patients. Patient concordance is an important issue. Once daily medicines and medicines with a low incidence of adverse effects are important, and there is also likely to be wider use of combination treatments, not just for hypertensive treatment but covering the totality of cardiovascular risk.

\section{REFERENCES}

1 National High Blood Pressure Education Program Working Group. Report on primary prevention of hypertension. Arch Intern Med 1993;153:186-208.

2 Martin MJ, Hulley SB, Browner WS, et al. Serum cholesterol, blood pressure and mortality: implications from a cohort of 361,662 men. Lancet 1986;ii:933-6.

3 Haffner SM, Lehto S, Ronnemaa T, et al. Mortality from coronary heart disease in subjects with type 2 diabetes and in nondiabetic subjects with and without prior myocardial infarction. N Engl J Med 1998;339:229-34.

4 Mokdad AH, Ford ES, Bowman BA, et al. Diabetes trends in the US: 19901998. Diabetes Care 2000;23:1278-83.

5 Collins R, Peto R, Macmahon S, et al. Blood pressure, stroke, and coronary artery disease. Part 2, Short-term reductions in blood pressure: overview of randomised drug trials in their epidemiological context. Lancet 1990;335:827-38.

6 Hansson L, Zanchetti A, Carruthers SG, et al. Effects of intensive bloodpressure lowering and low-dose aspirin in patients with hypertension: principal results of the hypertension optimal treatment (HOT) randomised trial. Lancet 1998:351:1755-62.

7 UK Prospective Diabetes Group. Tight blood pressure control and risk of macrovascular and microvascular complications in type 2 diabetes: UKPDS 38. BMJ 1998;317:703-13.

8 Verschuren WM, Jacobs DR, Bloemberg BP, et al. Serum total cholesterol and long-term coronary heart disease mortality in different cultures. Twenty-fiveyear follow-up of the seven countries study. JAMA 1995;274:131-6.

9 Brady AJB, Betteridge DJ. Prevalence and risks of undertreatment with statins. Br J Cardiol 2003; 10:218-9.

10 Primatesta P, Brookes M, Poulter NR. Improved hypertension management and control: results from the health survey for England 1998. Hypertension $2001 ; 38: 827-32$ 
11 EUROASPIRE I and II Group. Clinical reality of coronary prevention guidelines; a comparison of EUROASPIRE I and II in nine countries. Lancet 2001;357:995-1001

12 Primatesta $\mathbf{P}$, Poulter NR. Lipid concentrations and the use of lipid lowering drugs: evidence from a national cross sectional survey. BMJ 2000;321:1322-5.

13 Erhardt L, Hobbs FDR. Public perceptions of cardiovascular risk in five European countries: the REACT study. Int J Clin Pract 2002;56:638-44.

14 Hobbs FD, Erhardt L. Acceptance of guideline recommendations and perceived implementation of coronary heart disease prevention among primary care physicians in five European countries: the reassessing European attitudes about cardiovascular treatment (REACT) survey. Fam Pract 2002; 19:596-604.

15 British Cardiac Society, British Hyperlipidaemia Association, British Hypertension Society, British Diabetic Association. Joint British recommendations on prevention of coronary heart disease in clinical practice. Heart 1998;80(suppl 2):S1-29.

\section{DISCUSSION}

Question: There seems to be evidence that folic acid is beneficial $-0.8 \mathrm{mg}$, which is about three times what we normally eat-yet the information that this is probably a good thing for patients to eat is not getting out to general practice and to patients.

Professor Hobbs: In terms of public health policy I agree folic acid is important, but it is important for neural tube defect prevention among women who may become pregnant, rather than an important cardiovascular risk intervention. One of the problems with extrapolating from epidemiological studies, which has been the case for folic acid, is that it may not be borne out in actual trial evidence. The best example of this is hormone replacement therapy [HRT]: for years we have been promoting HRT to women at least partly as a cardiovascular protective agent on strong epidemiological evidence, and yet when the intervention trial was done to test that hypothesis it produced adverse effects in women, and has obviously modified our thinking on HRT. In terms of intervention, I think the evidence for folic acid is lacking in terms of cardiovascular risk reduction, as it is for any vitamin or mineral supplements in isolation.

Healthy living is what it is all about and the more we can advise successful ways of healthier living, the better it will be. But it was a bizarre thing to include folic acid in the polypill, compared to the other components that were being suggested. The main argument I would have against the polypill is the concept that a good public health policy is one that provides a little bit of benefit to everybody: those at relatively low risks are going to get benefit from that low intervention but it will undertreat those at high risk who require more aggressive intervention, and it will expose a significant proportion of people to some risk as well as adverse effects. I think the concept of combination treatment once patients are stabilised on a regimen is the right way forward, when you have not just identified what needs to be done but have also achieved a reasonable target for which risk factor needs to be intervened upon.

Question: Coming from Birmingham, I thought you might say something about ethnic issues and how we might target that particular group in a special way.
Professor Hobbs: This is a very important point and a difficult one because we probably do need special programmes for ethnic minority populations in the UK. Almost all of the attributable risk in ethnic minority populations, as indeed it is with different socioeconomic populations, is probably related to the presence of risk factors in much higher proportions and at greater elevated levels. Ethnic minority populations in the UK have much more prevalent rates of risk factors and their risk factors are more abnormal when present. That is what produces the greatly enhanced risk of the population. This reinforces the importance of identifying risk factors among risk populations and intervening appropriately. And that is also true of inner city populations. The different prevalence and intensity of risk factors probably explains $90 \%$ of the differences between different socioeconomic groups.

Question: I agree there are a lot of extra risk factors in this group and that they are high risk, but we need to be thinking laterally about different ways to manage them, because to see them in a 10 minute clinic may not be the best approach.

Professor Hobbs: Especially when you are speaking different primary languages, I think you are absolutely right. But that comes back to some of these bigger, wider issues. The first standard of the NSF is about the responsibility of government to produce a more informed public in an environment that is safer and more enriching. That is not something that medicine can deliver. That is only something we can deliver as a country. I think that Dr Roger Boyle has done a good job in widening the brief at the Department of Health, but many other departments in government don't even think about their wider responsibilities to the community. One thing that came up earlier with which I completely agree is this issue of generational issues in the UK-you could almost be heretical and say we should largely ignore the current adult population, they are doomed by their longstanding gene/environment interactions, and we should just concentrate on the next generation, the children who are going to grow up with an even higher prevalence of risk factors.

Question: Professor Barker's work shows quite clearly that if we have deprivation and these children live in utero in a state of malnutrition, when they come out into a world of fast foods they are metabolically not equipped to deal with it.

Professor Hobbs: I agree. There are all sorts of genetic reasons why we need to intervene aggressively now.

Professor Cowie: Picking up on one of those things, one of the big worries for the future is the rate of smoking among young women. Is there anything the health service can do, or is that something that the glossy women's magazines have more to do with?

Professor Hobbs: The social acceptability of smoking is a big issue. I think California and some other American states have got it right by banning smoking in public places. We want cigarettes to be really expensive, and so we don't want the EU allowing parallel imports. It is also scandalous that the EU still subsidises tobacco production. 